\title{
The prevelance of human papillomavirus (HPV) genotypes detected by PCR in women with normal and abnormal cervico-vaginal cytology
}

\author{
Fatma Beyazit ${ }^{1}$, Fatma Silan², Meryem Gencer ${ }^{1}$, Buket Aydin ${ }^{1}$, Baris Paksoy ${ }^{2}$, \\ Mesut A. Unsal ${ }^{1}$, Ozturk Ozdemir ${ }^{2}$ \\ ${ }^{1}$ Department of Obstetrics and Gynecology, Facultyof Medicine. Canakkale Onsekiz Mart University, Canakkale, Turkey \\ ${ }^{2}$ Department of Medical Genetics, Faculty of Medicine. Canakkale Onsekiz Mart University, Canakkale, Turkey
}

\begin{abstract}
Objectives: Cervical cancer is the second most common type of cancer for women worldwide with a great proportion proved to be related to human papillomavirus (HPV) infection. As infection with HPV is the strongest risk factor for cervical neoplasia, detection of HPV genotypes in cervical and vaginal specimens of women with normal and abnormal cytology seems to be of paramount importance in cervical cancer screening. The objective of the study is to evaluate the prevalence and HPV genotypes among women with normal or abnormal Pap smear tests.

Material and methods: This retrospective study was conducted in a tertiary care university hospital in western Turkey. A total of 201 patients in whom both HPV typing and Pap test was performed between 2012 and 2016 in our obstetrics and gynecology department were enrolled in this study. Clinical and laboratory data were obtained for all participants. Cervical smears of the patients were classified by the Bethesda system and HPV analyses were done using the polymerase chain reaction (PCR) method.

Results: This study included 201 women, 72 of whom had normal and 129 of whom had abnormal Pap smear results. HPV DNA was detected in 91 (45.2\%) of the 201 investigated women. Out of 72 patients with normal cervico-vaginal cytology, HPV positivity was detected in 35 (49\%) patients, whereas 33 (35\%) patients out of 94 with ASCUS, 18 (62\%) patients out of 29 with LSIL and $5(83 \%)$ patients out of 6 with HSIL had HPV positivity. Out of 35 HPV positive women that had normal pap test results, 25 (75\%) were found to have high risk HPV (HR-HPV) genotypes. In women with ASCUS, LSIL and HSIL, HR-HPV genotype rates were found to be $94 \%, 89 \%$ and $100 \%$ respectively. The most common identified HPV types were HPV58, HPV16, HPV31, HPV33, HPV11 and HPV35.

Conclusions: The frequency of HPV infection was found to be higher in our study compared to previous reports. Moreover, although HR-HPV genotypes were also detected in patients with normal cervical cytology, a majority of patients with HR-HPV genotypes were associated with abnormal cervical smear cytology including high rates of atypical squamous cells of undetermined significance, low-grade squamous intraepithelial lesion, and high-grade squamous intraepithelial lesion. Key words: Cervical cancer, Pap smear, squamous intraepithelial lesion
\end{abstract}

Ginekologia Polska 2018; 89, 2: 62-67

\section{INTRODUCTION}

Cervical cancer (CC) is the second most common cancer in women worldwide and the fourth-most common cause of death from cancer in women [1]. There is a large difference in terms of mortality and morbidity rates of CC in developing and developed countries because CC cases have been significantly reduced in developed countries due to the implementation of effective screening programmes [2]. Based on the difficulties in implementing cytology-based screening tools in developing countries like Turkey, the burden of disease remains very high, pointing to the need to engage and educate the broader public on prevention of CC [3].

Cervical cancer is a preventable disease because having a relatively long lead time and in general precancerous lesion slowly progress through recognizable stages before transforming into invasive disease. If the disease 
could be identified before progression to advanced stages, it can nearly almost be regarded as curable [4]. Today, by epidemiologic and molecular investigations, it is well established that a persistent oncogenic or so-called human papillomavirus (HPV) infection is the leading cause in the development of CC [5-7]. Nearly > 90\%_of all CCs are reported to be associated with this circular, double-stranded oncogenic virus [8].

HPV is an intraepithelial virus from the papillomavirus family that affects the human skin and the moist membranes that line the body and is passed between people through skin-to-skin contact. More than 100 different papilloma family members have been reported to exist, and in humans, these are responsible for a distinct variety of benign and malignant conditions. Although genital HPV types have been subdivided into high-risk (HR-HPV) types (frequently associated with invasive CC) and low-risk (LR-HPV) types (found mainly in genital warts), there is still no consensus on categorization of many HPV subtypes with low prevalence according to CC risk. Current epidemiologic data identifies $15 \mathrm{HPV}$ types as high-risk types $(16,18,31,33,35,39,45,51,52,56$, $58,59,68,73$, and 82$)$ and 12 types $(6,11,40,42,43,44,54,61$, $70,72,81$, and CP6108) as low-risk types [9]. Moreover, infection with two specific high-risk HPVs (HPV-16 and HPV-18) is reported to be linked with $90 \%$ of all uterine cervical cancers, and more than half of other anogenital tumors, and a small percentage of head and neck tumors [10].

Apart from HPV testing, cytology-based screening which is known as Papanicolaou-stained cervical smear (Pap smear or Pap test) can also be used for CC screening. Abnormal results in Pap test includes atypical squamous cells of undetermined significance (ASCUS), low-grade squamous intraepithelial lesion (LSIL), high-grade squamous intraepithelial lesion (HSIL), atypical glandular cells not otherwise specified (AGC-NOS) and squamous cell carcinoma [11]. Atypical glandular cells of undetermined significance (AGUS) term is eliminated to avoid confusion with ASCUS according to 2001 Bethesda system [11, 12]. Noncancerous conditions can also be found in Pap test including infections and inflammation. Although Pap test is acknowledged worldwide as being the most successful cancer screening test, HPV cotesting has markedly improved the ability to detect severe cervical dysplasia and provided better prevention against the development of CC. The objective of this research is to determine the frequency of distinct HPV genotypes in cervical discharge and its relation with cervico-vaginal cytology.

\section{MATERIAL AND METHODS Material}

This retrospective study was performed on 201 patients [median age 42 years (min-max: 20-75)] in whom both
HPV typing and Pap test was performed between 2012 and 2016 in obstetrics and gynecology department of Canakkale 18 Mart University, Turkey. After the approval from the same university's Institutional Ethics Board, we used the hospital's electronic database which contains multiple data fields on demographic, clinical and laboratory variables for patients. The study was conducted in accordance with the guidelines of the Helsinki declaration.

Women with intact uterus with distinct gynecologic complaints including post-coital bleeding, dyspareunia, lower abdominal pain with no past history of gynecologic malignancy were included in the present study. Exclusion criterias were as follow; women below age of 20 , current pregnancy, history of cervical conization and uterin prolapsus. Cervical smears were prepared by using cervical brush, fixed in $95 \%$ alcohol and stained by conventional methods. Cervical smears of the patients were examined under the microscope and classified by the Bethesda system [11] in the following categories: Normal, ASCUS, LSIL and HSIL.

\section{DNA isolation and HPV genotyping}

Total genomic DNA was extracted from cervicovaginal smear samples by using the commercial QIAamp Blood and Tissue Kit (QIAGEN Inc., Hilden, Germany) according to the manufacturer's protocol [13]. The target HPV genotyping was done after total genomic DNA isolation from cervicovaginal smear samples for each patient. Briefly HPV genotyping performed by the F-HPV typing ${ }^{\mathrm{TM}}$ multiplex PCR (Molgentix SL, Barcelona, Spain). The assay uses 16 specific primers amplifying within E6 and E7 regions of the HPV genome and permits the detection of 13 high risk genotypes $(16,18,31,33,35,39,45,51,52,56,58,59$ and 68$)$ and two low-risk genotypes ( 6 and 11). The presence of DNA of these HPV subtypes were detected by multiplex PCR amplification using type specific primers $[14,15]$.

For the multiplex PCR, in a total of $25 \mu \mathrm{L}$ reagent mixtures contained $5 \mu \mathrm{L}$ f-HPV PCR master mix that contained $5 \mathrm{U}$ hot start taq DNA polymerase enzyme, $15 \mu \mathrm{L}$ primers mix and $5 \mu \mathrm{L}$ target DNA were used for the amplification of target genes. The amplification conditions were; $96^{\circ} \mathrm{C}$ for $2 \mathrm{~min}$ for initial denaturation and holding phase cycles and in a total of thirty-five amplification cycles (ten and twenty-five tandem repeated sequences) were performed in the current genotyping procedure. Ten cycles included denaturation at $94^{\circ} \mathrm{C}$ for $30 \mathrm{~s}$, annealing at $64^{\circ} \mathrm{C}$ for $40 \mathrm{~s}$ and extension at $70^{\circ} \mathrm{C}$ for $30 \mathrm{~s}$ tandemly followed by twenty-five cycles included denaturation at $90^{\circ} \mathrm{C}$ for $30 \mathrm{~s}$, annealing at $60^{\circ} \mathrm{C}$ for $40 \mathrm{~s}$ and extension at $70^{\circ} \mathrm{C}$ for $30 \mathrm{~min}$. In the final phase, the temperature was maintained at $60^{\circ} \mathrm{C}$ for $30 \mathrm{~min}$ for polymerization of incomplete fragments. The PCR amplicons were separated and detected in terms of their size by using capillary electrophoresis. 
Table 1. Relationship between cytological screening of cervical Pap smears and Human Papillomavirus (HPV) test results

\begin{tabular}{|l|c|c|c|c|}
\hline & \multicolumn{2}{|c|}{ Human Papillomavirus (HPV) } & \\
\hline & Positive [n, \%] & Negative $[\mathbf{n}, \%]$ & Total $[\mathbf{n}, \%]$ & \\
\hline Normal & $35(49)$ & $37(51)$ & $72(100)$ & 0.016 \\
\hline ASCUS & $33(35)$ & $61(65)$ & $94(100)$ & $29(100)$ \\
\hline LSIL & $18(62)$ & $11(38)$ & $6(100)$ & \\
\hline HSIL & $5(83)$ & $1(17)$ &
\end{tabular}

ASCUS — atypical squamous cells of undetermined significance; HSIL — high-grade squamous intraepithelial lesion; LSIL — low-grade squamous intraepithelial lesion

\begin{tabular}{|l|c|c|}
\hline \multicolumn{3}{|c|}{ Table 2. Prevalence of human papillomavirus (HPV) types } \\
\hline HPV type & $\mathbf{n}$ & $\%$ \\
\hline HPV6 & 10 & 11 \\
\hline HPV 11 & 4 & 4.4 \\
\hline HPV 16 & 15 & 16.5 \\
\hline HPV 31 & 5 & 5.5 \\
\hline HPV 33 & 5 & 5.5 \\
\hline HPV 35 & 4 & 4.4 \\
\hline HPV 45 & 1 & 1.1 \\
\hline HPV 51 & 2 & 2.2 \\
\hline HPV 52 & 2 & 2.2 \\
\hline HPV 56 & 1 & 1.1 \\
\hline HPV 58 & 13 & 14.3 \\
\hline HPV 59 & 2 & 2.2 \\
\hline Mix & 27 & 29.6 \\
\hline Total & 91 & 100 \\
\hline
\end{tabular}

*Mix HPV types: $6 / 16 ; 6 / 58 ; 6 / 59 ; 6 / 56 / 59 ; 6 / 39 / 58 ; 11 / 16 ; 16 / 39 ; 16 / 52 ; 16 / 68$ $59 / 68 ; 18 / 51 ; 39 / 52 ; 51 / 58 ; 52 / 68 ; 58 / 59 ; 56 / 58$

\section{Statistical analysis}

Statistical analysis was done with SPPS version 19 (SPSS Inc., Chicago, IL, USA) software. Data were expressed as percentages of the mean or as genotype frequency. Chi-square test was used to compare categorical measurements between the groups. The statistical significance level was accepted as 0.05 .

\section{RESULTS}

Out of 201 women included in the present study, HPV DNA was detected in 91 (45.2\%) women. The median ages of HPV positive and negative subjects were 40 (min-max: 20-75) and 44 (min-max: 22-73) years respectively. According to cytological screening, 72 patients had normal cytologic findings, 94 patients had ASCUS, 29 had LSIL and 6 had HSIL. HPV positivity was detected in 35 (49\%) patients with normal cervico-vaginal cytology, whereas 33 (35\%) patients with ASCUS, 18 (62\%) patients with LSIL and 5 (83\%) patients with HSIL had HPV positivity (Tab. 1). HPV positivity was found to be related to abnormal cytologic test results $(p=0.016)$

The most common identified HPV types were HPV58, HPV16, HPV31, HPV33, HPV11 and HPV35. Twenty-seven (27.4\%) women had mixed HPV types. Table 2 shows all HPV types encountered in study population.

Women with positive HPV DNA was subdivided into two groups including HR-HPV [ $=77$ (84.6\%)] and LR-HPV $[n=14(15.4 \%)]$. Pap tests of women were evaluated according to these risk groups. Out of $35 \mathrm{HPV}$ positive women with normal Pap test results, 25 (75\%) had HR-HPV genotypes. In women with ASCUS, LSIL and HSIL, HR-HPV genotype rates were found to be $94 \%, 89 \%$ and $100 \%$ respectively (Tab. 3). According to HPV infection risk, cervical dysplasia (HSIL) rates were found to be elevated in HR-HPV subtype group $(p=0.047)$.

\section{DISCUSSION}

Our study population consisted of women with various genital complaints and nearly half of the study participants (45.2\%) presented HPV positivity which was significantly higher compared to studies from Turkey $[16,17]$ and several western countries $[18,19]$ and similar to some developing countries including Latin America and the Caribbean [20,21].

\begin{tabular}{|l|c|c|c|c|}
\hline \multicolumn{6}{|l|}{ Table 3. Cytological screening of cervical Pap smears according to HPV infection risks } \\
\hline & High-risk HPV [n, \%] & Low-risk HPV [n, \%] & Total [n, \%] & p \\
\hline Normal & $25(71)$ & $10(29)$ & $35(100)$ & 0.047 \\
\hline ASCUS & $31(94)$ & $2(6)$ & $33(100)$ & $18(100)$ \\
\hline LSIL & $16(89)$ & $2(11)$ & $5(100)$ \\
\hline HSIL & $5(100)$ & $0(0)$ &
\end{tabular}

ASCUS — atypical squamous cells of undetermined significance; HSIL — high-grade squamous intraepithelial lesion; LSIL — low-grade squamous intraepithelial lesion 
Apart from these elevated rates of HPV infection, we also found that HPV 16 and HPV 58 was associated with common abnormal cervical smear test results including high rates of ASCUS, LSIL and HSIL. These elevated rates of abnormal cytologic test results that has found to be linked with HPV positivity indicates that HPV is a major risk factor in the development of CC.

Globally CC is the third frequent cancer for woman following breast cancer and colorectal cancer [22]. It is a unique cancer because of being preventable in nature due to successful applications of cervical smear screenings. In developed countries where screening activities commonly applied success rates are particularly impressive whereas in developing countries CC still causes significant number of deaths [23]. According to Turkey cancer control programme, $\mathrm{CC}$ is the $10^{\text {th }}$ most frequently observed cancer in women following breast, thyroid, colorectal, uterus corpus, lung, stomach, ovary, non-Hodgkin lymphoma and brain cancers [3]. Although it can be speculated that CC does not represent a serious threat in Turkey compared with other cancers, a global CC screening program with cervical smear test has been applied by department of cancer of Ministry of Health since 1992 [24]. In accordance with global scientific trends, national screening programme for CC was changed after two decades and HPV testing was implemented as a primary screening for CC [23]. According to the Renewed National Cervical Screening program, HPV test once in 5 years and smear evaluation in HPV positive cases is recommended to every women between ages of 30-65 [3]. In this context, it is important for centers to present their own results to the scientific community and exchange knowledge and practice on preventing CC development.

The results of the present study demonstrated that HPV16, HPV 58, HPV6, HPV31, HPV33 and HPV11 were the six most prevalent types in our study population. In a study by Ari et al. [25] the most common HPV types that infect the genital area was reported to be HPV16 and HPV6. In another study from Turkey, Yavuzer and colleagues [26] investigated the prevalence of HPV DNA and reported the distribution of HPV types among women with cervical cancerous and precancerous lesions. The authors stated that the most common three HPV types were as follows; HPV6, HPV11 and HPV 31. Analysis of existing studies in different countries regarding the types of HPV infection in abnormal cervical scrapes also demonstrated similar results. Finan et al. [27] reported that by using the consensus primer set MY09/MY11 HPV types $6,11,16,18$ and 33 were the most common detected HPV genotypes in endocervical and vaginal scrapes of women with abnormal cytology and also in those with normal cytology. In a study by Antonishyn et al. [28] it was found that the most commonly encountered HPV genotype in patients with cervical intraepithelial neoplasia grade 2 or
3 was HPV16 (46.7\%) which was followed by HPV-31 (14.7\%) and HPV-18 (3.9\%). Apart from these studies, a recent study from Africa in which the prevalence of HPV infection, human immunodeficiency virus (HIV) infection, and cervical cytologic abnormality rates were investigated in women who were admitted to a sexually transmitted infections clinic demonstrated that most common HPV genotypes were 52,58 and 16 . Moreover all of the HIV positive women were reported to be co-infected with HPV [29].

The incorporation of HPV cotesting into cervical screening programmes has undoubtedly improved the ability to detect cervical dysplasia/neoplasia and provided better prevention against CC. This algorithm is also associated with somewhat worsened specificity and an elevated risk for not detecting new glandular lesions. In a study by Al-Awadhi and colleagues [30] the prevalence and type specific distribution of HPV in women with normal cervical cytology was investigated. The authors reported relatively low rates $(2.4 \%)$ of HPV positivity in their patient population and these low rates were attributed to unique sociodemographic and sexual behaviour characteristics of the country. In a study from Turkey, although HPV DNA was found to be positive in $20 \%$ of the women with normal cervical cytology, it was positive in $36 \%$ of women with abnormal cervical cytology [17]. The reason for this low HPV rates in women with abnormal cytology explained by technical or sampling biases. In our study, we found that high-risk HPV types were associated with increased cervical dysplasia rates in cervico-vaginal smears and $32.5 \%$ (25/77) of HR-HPV positive women had normal Pap tests. These patients with normal Pap tests and HR-HPV types must be regularly followed up in accordance with international guidelines. This is because of the fact that women with HR-HPV positivity and negative Pap smear tests for intraepithelial lesions are at a high risk of having serious HPV induced lesions in the future [31]. Delayed follow-up may have jeopardized these patients at risk for serious epithelial changes. For this reason, in women with slight cell alterations (such as ASCUS and LSIL) follow-up testing is commonly recommended. In women with HSIL, colposcopy with endocervical sampling has been considered the best management [32]. Increased cervical dysplasia rates observed in HR-HPV infections lend support to the suggestion that differences in HPV type may indeed correlate with differences in biological potential and risk for invasion in LSIL and HSIL lesions.

This study showed that the HPV infection rate in Turkey is more common compared with previous studies. Moreover, based on the presence of high rates of HR-HPV genotypes even in women with normal cervico-vaginal cytology necessitates more close follow-up with immediate reference to colposcopy. Therefore, despite the fact that current preventive 
vaccines significantly reduce but not eliminate the risk of cervical cancers and other premalignant diseases related to HPVs, there is a need for effective immunological treatment of HPV and related disorders.

\section{Limitations}

We recognize that there are limitations to our study design. First, our study population is relatively small and limited to the patients that were admitted to a single center, therefore conclusions from the present study could not be generalized further. Moreover, despite this limited number of subjects, we found significant differences between subgroup analysis. Second, the retrospective design of the study did not permit us to adequately analyze the prognostic variables and management. However, since the goal of the present study was to examine the relationship between HPV and cervical cytological associations, there was no way to overcome the limitations which necessarily accompany a retrospective design. Third, although universal colposcopy is not part of standard clinical care, it would be noteworthy if colposcopic examination with endocervical assessment had taken place.

\section{CONCLUSIONS}

1. HPV DNA testing with cervicovaginal smear analysis can improve the detection rate of cervical intra epithelial lesions.

2. HPV infection is more prevalent than expected among women in Turkey, which indicates higher attention to prevention and screening in the gynecology and obstetrics clinics.

3. High risk HPV types have great value in determining high grade of cervical intraepithelial neoplasia and subsequent cervical cancer development.

\section{Conflict of interest}

None declared.

\section{Ethical approval}

Ethical approval was obtained.

\section{REFERENCES}

1. Ferlay J, Shin HR, Bray F, et al. Estimates of worldwide burden of cancer in 2008: GLOBOCAN 2008. Int J Cancer. 2010; 127(12): 2893-2917, doi: 10.1002/ijc.25516, indexed in Pubmed: 21351269.

2. Catarino R, Petignat $P$, Dongui $G$, et al. Cervical cancer screening in developing countries at a crossroad: Emerging technologies and policy choices. World J Clin Oncol. 2015; 6(6): 281-290, doi: 10.5306/wjco. v6.i6.281, indexed in Pubmed: 26677441.

3. Özmen V, Dağoğlu N, Dede I, et al. Turkish Ministry of Health, 2nd Turkish Medical General Assembly Clinical Oncology Study Group Report. J Breast Health. 2016; 12(1): 9-17, doi: 10.5152/tjbh.2015.2869, indexed in Pubmed: 28331725.

4. Ghosh S, Seth S, Paul J, et al. Evaluation of Pap smear, high risk HPV DNA testing in detection of cervical neoplasia with colposcopy guided or conventional biopsy as gold standard. Int J Healthcare Biomed Res. 2014; 2(2): 192-197.
5. Wardak S. Human Papillomavirus (HPV) and cervical cancer. Med Dosw Mikrobiol. 2016; 68(1): 73-84, indexed in Pubmed: 28146625.

6. Zhang $\mathrm{L}$, Bi $\mathrm{Q}$, Deng $\mathrm{H}$, et al. Human papillomavirus infections among women with cervical lesions and cervical cancer in Eastern China: genotype-specific prevalence and attribution. BMC Infect Dis. 2017; 17(1): 107, doi: 10.1186/s12879-017-2223-1, indexed in Pubmed: 28143439.

7. Sasaki Y, Iwanari O, Arakawa I, et al. Cervical Cancer Screening With Human Papillomavirus DNA and Cytology in Japan. Int J Gynecol Cancer. 2017; 27(3): 523-529, doi: 10.1097/IGC.0000000000000898, indexed in Pubmed: 27997455.

8. Fernandez AF, Rosales C, Lopez-Nieva P, et al. The dynamic DNA methylomes of double-stranded DNA viruses associated with human cancer. Genome Res. 2009; 19(3): 438-451, doi: 10.1101/gr.083550.108, indexed in Pubmed: 19208682.

9. Abreu ALP, Souza RP, Gimenes F, et al. A review of methods for detect human Papillomavirus infection. Virol J. 2012; 9: 262, doi: 10.1186/1743422X-9-262, indexed in Pubmed: 23131123.

10. Agorastos T, Chatzistamatiou K, Katsamagkas T, et al. HERMES study group. Primary screening for cervical cancer based on high-risk human papillomavirus (HPV) detection and HPV 16 and HPV 18 genotyping, in comparison to cytology. PLoS One. 2015; 10(3): e0119755, doi: 10.1371/journal.pone.0119755, indexed in Pubmed: 25793281.

11. Solomon D, Davey D, Kurman R, et al. Forum Group Members, Bethesda 2001 Workshop. The 2001 Bethesda System: terminology for reporting results of cervical cytology. JAMA. 2002; 287(16): 2114-2119, indexed in Pubmed: 11966386.

12. Nayar R, Wilbur DC. The Bethesda System for Reporting Cervical Cytology: Definitions, Criteria, and Explanatory Notes, ed 3. Springer, New York 2015.

13. Lin WM, Ashfaq R, Michalopulos EA, et al. Molecular Papanicolaou tests in the twenty-first century: molecular analyses with fluid-based Papanicolaou technology. Am J Obstet Gynecol. 2000; 183(1): 39-45, doi: 10.1067/mob.2000.105734, indexed in Pubmed: 10920306.

14. Hesselink AT, Berkhof J, van der Salm ML, et al. Clinical validation of the HPV-risk assay, a novel real-time PCR assay for detection of high-risk human papillomavirus DNA by targeting the E7 region. J Clin Microbiol. 2014; 52(3): 890-896, doi: 10.1128/JCM.03195-13, indexed in Pubmed: 24391196.

15. Zou R, Xie W, Wang H, et al. Establishment and Application of a Method for High-Risk Human Papillomavirus Genotyping in Cervical Cancer Tissue. Clin Lab. 2016; 62(6): 1075-1085, indexed in Pubmed: 27468570.

16. Tunç SY, Onan MA, Turp AB, et al. Prevalence and types of cervical human papillomavirus among Turkish women and its relationship with demographic factors in a gynecology outpatient clinic. Eur J Gynaecol Oncol. 2016; 37(1): 53-58, indexed in Pubmed: 27048110.

17. Dursun $\mathrm{P}$, Senger SS, Arslan H, et al. Human papillomavirus (HPV) prevalence and types among Turkish women at a gynecology outpatient unit. BMC Infect Dis. 2009; 9: 191, doi: 10.1186/1471-2334-9-191, indexed in Pubmed: 19948015.

18. Tricco AC, $\mathrm{Ng} \mathrm{CH}$, Gilca V, et al. Canadian oncogenic human papillomavirus cervical infection prevalence: systematic review and meta-analysis. BMC Infect Dis. 2011; 11: 235, doi: 10.1186/1471-2334-11-235, indexed in Pubmed: 21892939.

19. Dunne EF, Unger ER, Sternberg M, et al. Prevalence of HPV infection among females in the United States. JAMA. 2007; 297(8): 813-819, doi: 10.1001/jama.297.8.813, indexed in Pubmed: 17327523.

20. Almonte $M$, Albero $G$, Molano $M$, et al. Risk factors for human papillomavirus exposure and co-factors for cervical cancer in Latin America and the Caribbean. Vaccine. 2008; 26 Suppl 11: L16-L36, doi: 10.1016/j. vaccine.2008.06.008, indexed in Pubmed: 18945400.

21. Deluca GD, Basiletti J, González JV, et al. Human papilloma virus risk factors for infection and genotype distribution in aboriginal women from Northern Argentina. Medicina (B Aires). 2012; 72(6): 461-466, indexed in Pubmed: 23241287.

22. McGraw SL, Ferrante JM. Update on prevention and screening of cervical cancer. World J Clin Oncol. 2014; 5(4): 744-752, doi: 10.5306/wjco. v5.i4.744, indexed in Pubmed: 25302174.

23. Nour NM. Cervical cancer: a preventable death. Rev Obstet Gynecol 2009; 2(4): 240-244, indexed in Pubmed: 20111660.

24. Açikgöz A, Ergör G. Cervical cancer risk levels in Turkey and compliance to the national cervical cancer screening standard. Asian Pac J Cancer Prev. 2011; 12(4): 923-927, indexed in Pubmed: 21790227.

25. Arı M, Döğer FK, Kırdar S, et al. Cervical Biopsy, Smear Evaluation and Comparison of Human Papilloma Virus Subtypes Result. Meandros Med Dent J. 2016; 17(1): 17-21, doi: 10.4274/meandros.2582. 
26. Yavuzer D, Karadayı N, Erdağı A, et al. typing with PCR in cervical cancerous and precancerous lesions. Kartal EAH Med J. 2009; 20(1): 1-6.

27. Finan $\mathrm{RR}$, Irani-Hakime $\mathrm{N}$, Tamim $\mathrm{H}$, et al. Detection of human papillomavirus (HPV) genotypes in cervico-vaginal scrapes of women with normal and abnormal cytology. Clin Microbiol Infect. 2001; 7(12): 688-692, doi: 10.1046/j.1469-0691.2001.00339.x, indexed in Pubmed: 11843911.

28. Antonishyn NA, Horsman GB, Kelln RA, et al. The impact of the distribution of human papillomavirus types and associated high-risk lesions in a colposcopy population for monitoring vaccine efficacy. Arch Pathol Lab Med. 2008; 132(1): 54-60, doi: 10.1043/1543-2165(2008)132[54:TIOTDO12.0.CO;2, indexed in Pubmed: 18181674.

29. Blossom DB, Beigi RH, Farrell JJ, et al. Human papillomavirus genotypes associated with cervical cytologic abnormalities and HIV infection in Ugandan women. J Med Virol. 2007; 79(6): 758-765, doi: 10.1002/jmv.20817, indexed in Pubmed: 17457908.
30. Al-Awadhi R, Chehadeh W, Kapila K. Prevalence of human papillomavirus among women with normal cervical cytology in Kuwait. J Med Virol. 2011; 83(3): 453-460, doi: 10.1002/jmv.21981, indexed in Pubmed: 21264866.

31. Banna NEl, Eyd GAl, Saeed R. High-risk human papillomavirus infection among women with pap smear tests negative for intraepithelial lesions or malignancy. Int J Med Public Health. 2014; 4(1): 102, doi: 10.4103/2230-8598.127168.

32. Stenvall H, Wikström I, Backlund I, et al. Accuracy of HPV testing of vaginal smear obtained with a novel self-sampling device. Acta Obstet Gynecol Scand. 2007; 86(1): 16-21, doi: 10.1080/00016340601033667, indexed in Pubmed: 17230283. 